\title{
PERFORMANCE ANALYSIS OF EFFECT RATE OF CROSS LAYER BASED INTRUSION DETECTION FOR WIRELESS LAN
}

\author{
Dr. Vinod Kumar \\ Assistant Professor \\ Department of Computer Science \\ Dev Samaj College for Women \\ Ferozepur City \\ Vinodkumarkamboj@gmail.com
}

\section{ABSTRACT}

Wireless ad-hoc networks are vulnerable to various kinds of security threats and attacks due to relative ease of access to wireless medium and lack of a centralized infrastructure. Security is an alarming concern, as everything being transmitted is available in the air. The current paper deals with Study of effect of rate on performance of cross layer based intrusion detection for WLAN reflects the significance of cross layer technique in detecting intruder on WLAN. Exploiting the information available across different layers of the protocol stack by triggering multiple levels of detection enhances the accuracy of detection. We validate our design through simulations and also demonstrate lower occurrence of false positives

\section{KEYWORDS}

Access protocols , Ad hoc networks , Availability Communication system security, Computer crime Cross layer design, Intrusion detection, Mobile ad hoc networks , Network topology , Wireless networks, Receiver Signal Strength (RSS); Time Taken for RTSCTS Handshake (TT); Radio Frequency (RF).

\section{INTRODUCTION}

While deploying a wireless LAN makes complete buissness sense for an organization care must be paid to properly lock the wirelss network down so that the network traffic, which oftern contains buissness -critical information from being revealed to or modified by unauthorized people. The wireless LAN infrastructure should be secure and security must be augmented with advanced algorithms. A wireless network is not as secure as compare the wired network because the data is transferred on air so any intruder can use hacking techniques to access that data. Indeed it is difficult to protect the data and provide the user a secure information system for lifetime. An intrusions detection system aim to detect the different attacks against network and system. An intrusion detection system should be capable for detecting the misuse of the network whether it will be by the authenticated user or by an attacker. They detect attempts and active misuse either by legitimate users of the information systems or by external.[1] The aim of intruder is to gain the access of the privileges. Generally this show that intruder want information which is protected.

\section{DETECTION SYSTEM}

Inevitably, the best intrusion prevention system will fail. A system's second line of defense is intrusion detection, and this has been the focus of much research in recent years. [2-7].

\author{
Mrs. Ritika Narang \\ Assistant Professor \\ Department of Computer Science \\ Govt Bikram College of Commerce \\ Patiala
}

\section{Types of intrusion detection systems}

There are two types of intrusion detection systems.

1. Network Based Intrusion Detection system (NIDS) which resides on network.

2. Host Based Intrusion Detection system (HIDS) which resides on host i.e. computer system. [811]

\section{Network Based intrusion detection system (NIDS)}

Network based intrusion detection system resides on network. It exists as software process on hardware system. It change the network interface card (NIC) into promiscuous mode, i.e. the card passes all traffic on the network to the NIDS software. The software includes the rules which are used to analyze the traffic. It analyzes the incoming packets against these rules to determine the signature of the attacker, whether this traffic signature is of any attacker or not. If it is of interest then events are generated [1,3]. The data source to NIDS is raw packets. It utilizes a network adapter which is running in promiscuous mode to monitor and analyze the network. There are four common techniques to identify attack.

a) Frequency or threshold crossing.

b) Correlation of lesser events.

c) Statistical anomaly detection.

d) Pattern, expression or byte code matching.

NIDS is not limited to read all the incoming packets only. But also learn the valuable information on outgoing traffic. With this feature the attacker form inside the monitored network are identified.

\section{Host Based Intrusion detection system (HIDS)}

Host based IDS are embedded on host computer. It exists as a software process on a system. So it examines the log entries in system for specific information. It identifies the new entries and compares them to pre configured rules. It also works on rule based, if the entry match to the rule then it will generate alarm that this is not legal user.

\section{Anomaly based detection}

Anomaly detection attempts to model the normal behavior. Any occurring event which violates this model behavior is reflecting to be suspicious. It aim is to detect the patterns that do not conform normal behavior. The 
pattern that does not conformed as normal are called as anomalies [12-13].

\section{Misuse based detection}

The equations are an exception to the prescribed specifications of this template. You will need to determine whether or not your equation should be typed using either the Times New Roman or the Symbol font (please no other font). To create multileveled equations, it may be necessary to treat the equation as a graphic and insert it into the text after your paper is styled.

\section{CROSS LAYER BASED TECHNIQUE}

Cross layer based technique is used to make decision that whether there is an attacker or not by combining the result of two or more layer in TCP protocol. [12-13]

\section{A) Monitoring Received Signal Strength (RSS)}

A measure of energy which is observed by the physical layer at the antenna of the receiver is called as Received signal strength (RSS). In IEEE 802.11 networks, while performing MAC clear channel measurement and in roaming operations, the RSS indication value is used. The radio frequency (RF) signal strength can be measured through absolute (decibel mill watts $-\mathrm{dBm}$ ), or relative (RSSI) manner. Exact RSS value from sender to receiver is not easy to assume as mention above. To assume exact value of RSS the attacker has to be present on the same location which is not possible. The radio equipment used by the receiver have to be same for identify exact value of RSS. Moreover there should be same level of reflection, refraction, and interface. Even if the sender is fixed, RSS value seems to vary a little and it is proved that it is almost not possible to guess. This restricts the attacker from using the radio equipment to spoof the RSS clearly by the receiver. A dynamic profile is build of the computer node which are communicating depend upon the RSS value from a server. Any sudden or unusual changes can be marked as doubtful activity which indicates the possible session of hijacking attack. Reason why one call RSS profile dynamic is because during every session it is build again and keep on updating. Any sudden changes in the RSS dynamic profile can be marked as doubtful activity with a higher confidence level because BSs are generally immobile. On the other hand, if the MS is mobile, then its respective RSS values will vary quickly which can be observed by the server. Therefore the uncertainty of the wireless medium can be used in the favor of intrusion detection, where the attacker is unable to know what RSS values to spoof. Therefore it is effective for the session hijacking attacks and it does not need any additional bandwidth consumption.

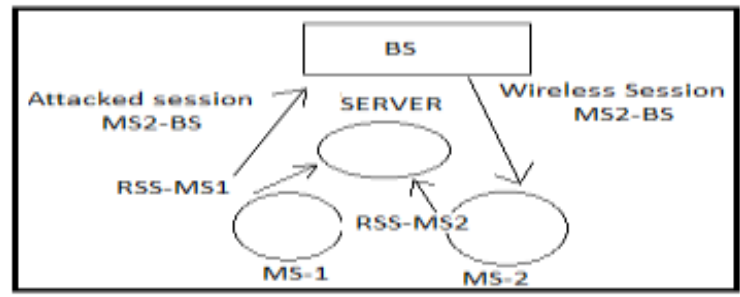

Figure 1 Received signal strength (RSS)

For example, based on the observed RSS values at the server it can develop a dynamic RSS profile for both MS2 and BS when a valid MS2 has an active session with a BS (Refer Fig.1). If a attacker MS1 hijacks MS2 through isolating from the network and spoofing its MAC address then the server will pick up the abrupt changes in the RSS profile of MS2's MAC and gives an alert signal. Since they depend on the MS1's actual location, radio equipment and surrounding environment the RSS values for the MS2's MAC address will change.

In another situation, if the attacker MS1 spoofs the base station BS then it will also get detected as the dynamic RSS profile for the BS undergoes sudden variations. Therefore this mechanism gives detection for both session hijacking and man-in-the-middle attacks which is targeted at either MSs or BSs.

\section{B) Monitoring Time Taken For RTS-CTS Handshake}

Virtual carrier sensing is created using RTS-CTS which makes the transmission of data frames possible without collision. The successful delivery of the CTS frame from the receiver shows that the receiver is received the senders RTS frame successfully and ready for receiving the data. The time taken to complete the RTS-CTS handshake between itself and receiver i.e. TT can be examined by the sender. This is the total time taken for the RTS frame to travel from the sender to receiver and also for the CTS frame to send an acknowledgement. RTS-CTS handshake is free from collisions with any network node.

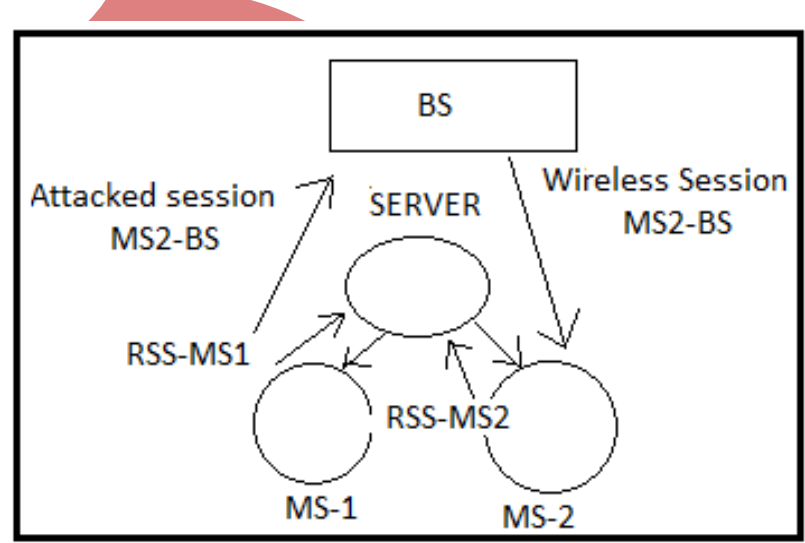

The TT values for a fixed transmission rate are not affected because the size of RTS and CTS frames are fixed and makes the TT between two nodes as an unspoofable parameter. So this cannot be easily guessed by an attacker when tracking the waves. Since it is calculated by the sender of the RTS-CTS handshake it is also protected from snooping. Since it is a measurement related to the entity measuring, the attacker should be exactly at the same location as the sender. Also the attacker should use the same radio equipment with the same attenuation and antenna gain. In order to predict the values of TT between the sender and receiver as measured by the sender, the attacker should receive the radio waves after the same number of reflections and refractions. It can also be calculated without any particular computational.

From the intrusion detection point of view, a mechanism which is used to detect the session hijacking attacks uses the quick and sudden changes in the TT betoneen the two nodes. Server can measure the time elapsed betoneen when it detects RTS frame from the sender to receiver and when it detects a return CTS from the receiver back to the sender i.e. TT. For understanding, this time can be represented as,

$\mathrm{TT}=\mathrm{TT}_{\mathrm{M}}-\mathrm{TT}_{\mathrm{S}-\mathrm{R}}-\mathrm{TT}_{\mathrm{M}-\mathrm{S}}$ 
Where ,

TT $_{S-R}$ - time taken for a RTS frame to cover the distance between the sender and the server, $\mathrm{TT}_{\mathrm{M}-\mathrm{S}}$ time taken for a frame to cover the distance between the server and the receiver, RTS, $\mathrm{TT}_{\mathrm{M}}$ time taken for a handshake to complete between a sender and receiver as observed by the server. CTS-RTS. But the server does not know these actual values. Monitoring observed TT values at the server provides reliable passive detection mechanism for session hijacking attacks since TT is an unspoofable parameter related to its measuring entity. Also this cannot be guessed because its exact value depends on

1) The position of the receiver and the server

2) The distance between the server and receiver

3) The environment around the receiver and the server.

This is a property which cannot be measured or spoofed by an attacker when tracking the network traffic or using a specialized radio equipment. It has been propose [13] that changes in TT between two communicating nodes can be observed by a passive server and the sudden variations are marked as suspicious. This helps to detect the attacker who tries to take over a receiver's session by isolating it off the network and spoofing its MAC address. On the other hand, the RTS-CTS handshakes which originates from the receiver is used to detect the session hijacking attacks which aims the sender. For example, the server can develop a dynamic RSS profile which gets constantly updated per session and it calculates the TT for every RTS -CTS handshake from both MS2 and BS when a valid MS2 has an active session with a BS (Refer Fig.2). If an attacker MS1 hijacks MS2 through spoofing its MAC address then the server will observe abrupt changes in the TT for MS2 and gives an alert signal. Also to detect the man-in-themiddle attacks against BS, TT values from RTS -CTS handshakes between MS2 and BS which originates from MS2 can be registered by the server in the MS2's profile. The Server executes the following algorithm, to detect the attackers.

\section{Detection Algorithm}

\section{. Step 1: Server measures RSS}

Step 2: Server measures TT

Step 3: Server calculates the weight $\mathrm{W}$ as

$W=W 1 . \sigma_{R S S}+W 2 . \sigma_{T T}$

Where $\sigma_{\mathrm{RSS}}=$ Variation of ${ }_{\mathrm{RSS}}$ and $\sigma_{\mathrm{TT}}=$ variation of ${ }^{\mathrm{TT}}$ $\mathrm{w} 1$ and $\mathrm{w} 2$ are two constants. This technique has been successfully applied for intrusion detection in mobile adhoc network. [13] The present study aims the application of technique proposed [13] for WLAN system to study the effect of rate on the performance as well as comparison with existing techniques.

\section{EXPERIMENTAL ASPECT IN PRESENT STUDY}

The following hardware/software platform has been used to conduct the proposed study:

1. Hardware platform
INTEL CORE i5 $\mathrm{n}$ series processor

\section{Software platform}

LINUX OS

\section{Ns 2 simulator}

The number of wireless network devices will soon surpass the number of wired devices, and the amount of research in the area of wireless networking is increasing at a similar rate [9]. Wireless research often involves a testbed implementation and/or a simulation study. Network simulators allow researchers to analyze the behavior of these wireless devices at every level. As a result, these simulations are capable of producing very large amounts of data. The simulation community has made available many types of scripts (e.g., tracegraph [14]) to parse and analyze this output data, but visualization of the data is needed to further aid understanding of the output. A good visualization package is important, because the human visual system is unrivaled in pattern recognition and offers the ability to process large amounts of data quickly and clearly [3]. Visualization adds to the understanding gained via statistical analysis. As one show in this paper, certain erroneous network behaviors could go undetected without visualizations.

The Network Simulator 2 (NS-2) is a popular and poonerful simulation environment, and the number of NS-2 users has increased greatly in recent years. Although it was originally designed for wired networks, NS-2 has been extended to work with wireless networks, including wireless LANs, mobile ad hoc networks, and sensor networks.

\section{RESULTS AND DISCUSSION}

In order to test the protocol, the NS2 simulator is used. The experimental consist of 1 wired node, 1 base station and 4 master stations with one server. One compares our proposed cross-layer based intrusion detection technique with the Radio Frequency Fingerprinting (RFF) technique. The fig. 3 shows the snapshot of experimental setup for the present study

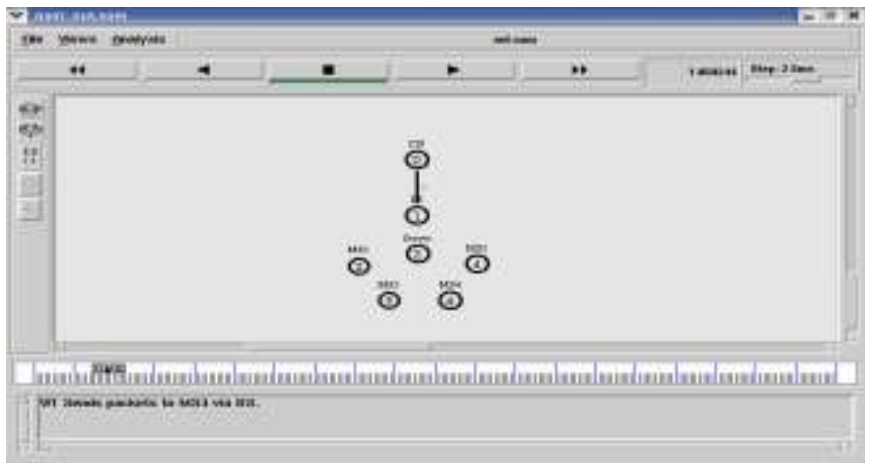

\section{Effect of Varying attack Rates}

In the first experiment, the attack traffic rate is varied as $50,100,150,200$ and $250 \mathrm{~kb}$. Figure 4 shows the rate vs delivery ratio of our cross-layer technique and RFF. From the figure, one can see that packet delivery ratio is more in cross-layer scheme when compared with RFF scheme. 


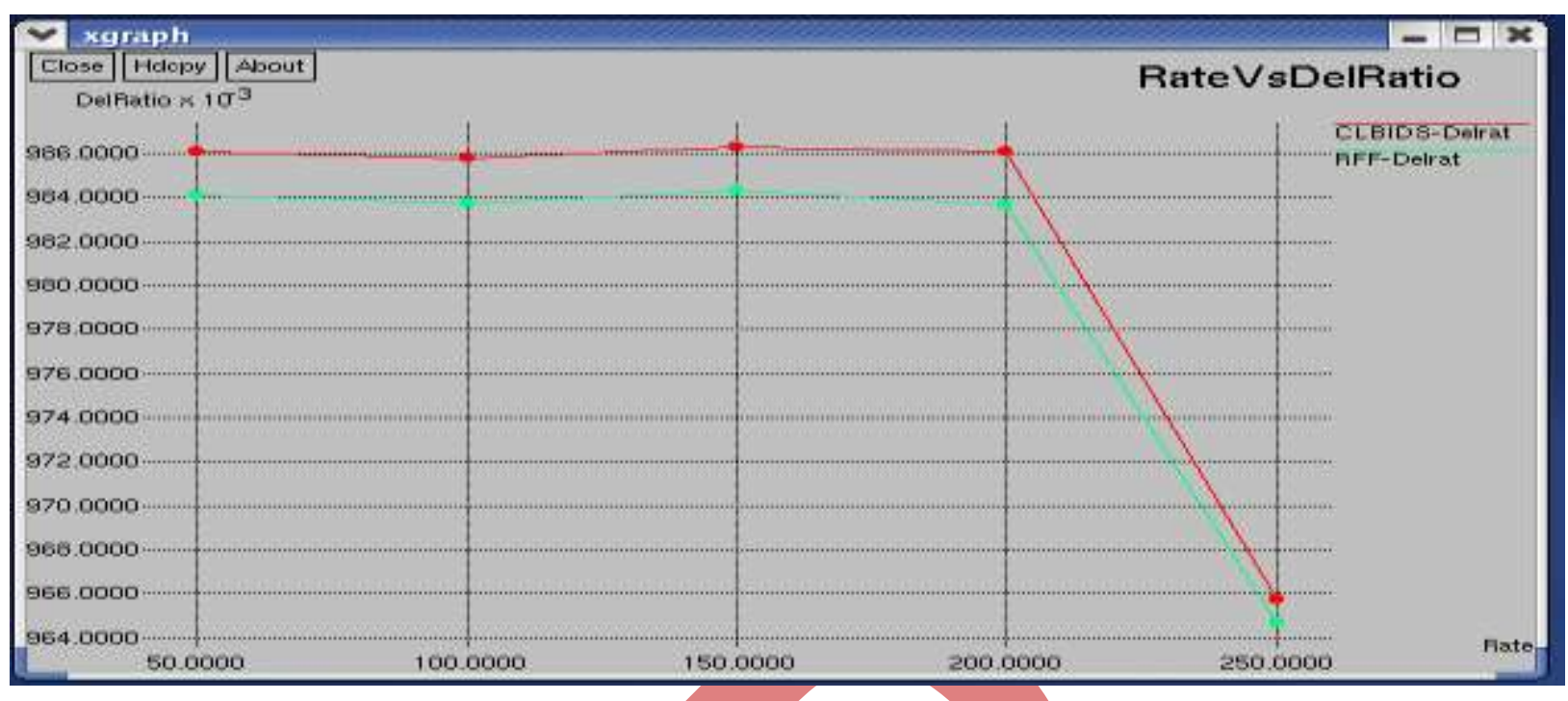

Figure shows the false positive rate of our cross-layer technique and RFF. From the figure, one can observe that our cross-layer scheme attains low false positive rate, when compared with RFF scheme, since it accurately detects the intrusion.

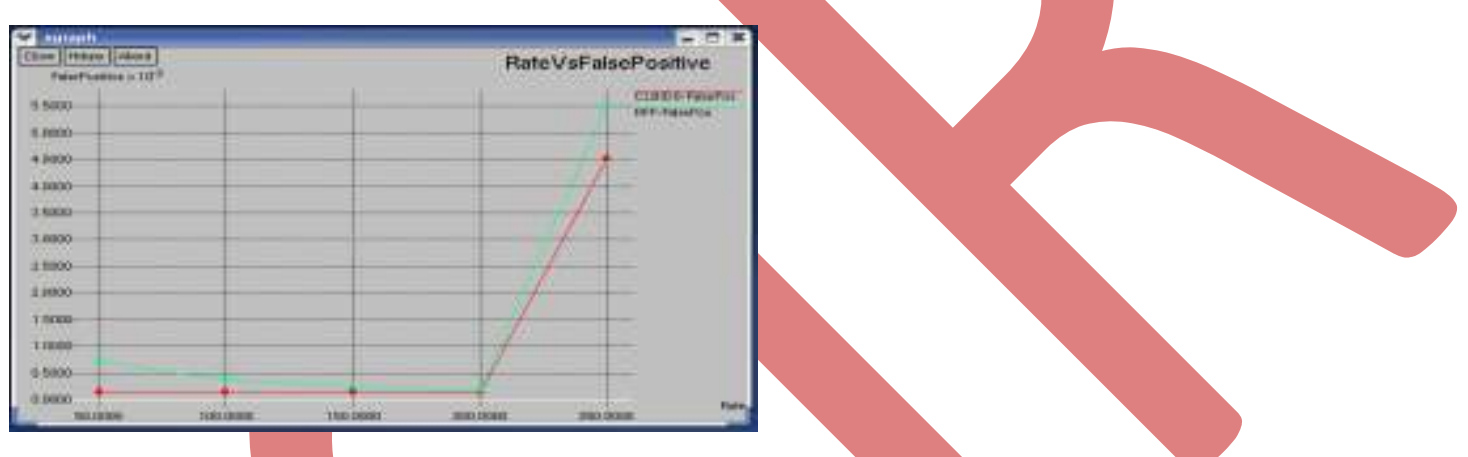

The purposed technique is based on cross layer, where two layers has been used i.e physical layer and MAC layer. On physical layer one compute the RSS value, which is evaluated by the receiver at destination node.RSS value, is computed by the omnidirectional antenna at receiver node. Second on MAC layer RTS/CTS handshake time is computed. RTS/CTS is used to avoid collision on network. The combine result value is submitted to the server, which becomes the threshold value Dth and when a new session is made the server will compare the current RSS and RTS/CTS time taken with the previous value Dth. If that value is less than previous value then it is an attacker. RSS value depends upon the type of antenna being used as onell as the reflection, refraction and interference. If an attacker what to pretend as the authenticated user by accessing its MAC address, but using this technique the attacker is unable to identify the RSS value because he may not be using same antenna and even there is not same reflection, refraction and interference. So he is unable to know the exact value.

RTS/CTS fame make the transmission of packets without collision .RTS/CTS time taken is computed by the sender. A successful delivery of CTS frame from receiver to sender shows that RTS frame is delivered to the receiver. RTS/CTS frame has a fixed transmission rate. This makes TT parameter unspoofable, so the attacker is unable to detect TT parameter. As describe in above cases it is difficult for an attacker to access the session, because only with MAC address he can't access the session, the server is computing RSS and RTS/CTS Time Taken (which is not exactly as the authenticated user). This shows that this technique will reduce the chance of intrusion. Moreover due to combine the result of two layers the false positive rate is also reduced.

\section{CONCLUSION}

In the scenario there are 1 wired node, 1 base station and 4 master stations (1 as server). The transmission rate is set to $100-400 \mathrm{~m}$. The antenna used here is omnidirectional, with two way propagation. There are two domains with one cluster in each, 1 node in first cluster and 6 in the second cluster. When the simulation starts the RSS value and RTS/CTS handshake time is captured. The threshold value is taken by the packet size plus the channel idle time. Then the procedure to check the attacker is started where one get the delta RSS, TT values by subtracting it from current values form the previous values. Both these values are evaluated by multiplying two oneight parameters $\mathrm{W} 1$ and $\mathrm{W} 2$. Then the combine result of these is checked against the Dth(threshold value). If it is less than the Dth value then that person is an attacker. It is concluded that packet delivery ratio is more in cross-layer scheme when compared with RFF scheme and cross-layer scheme attains low false positive rate, when compared with RFF scheme, since it accurately detects the intrusion. The simulation result shows that the CLBIDS technique is better technique then the RFF technique for WLAN system. 


\section{FUTURE WORK}

The effect of other dominant performance enhancing parameters will be incorporated in future for efficient intrusion detection system in the wireless domain. The future scope of this technique is one can implement this technique in many wireless hardware devices. It can be implemented in network where the decision is made whether to accept that attacker data for a destination node by computing RSS and TT values.

\section{IMPACT OF STUDY}

Wireless mesh networking has been a cost-effective technology that provides wide-coverage broadband wireless network services. They benefit both service providers with low cost in network deployment, and end users with ubiquitous access to the Internet from anywhere at anytime. Hoonever, as wireless mesh network (WMN) proliferates, security and privacy issues associated with this communication paradigm have become more and more evident and thus need to be addressed. The present study will be useful to provide a good foundation to implement real time detection.

We are thankful to Dr. Jatinder Singh (Dean and Professor, Computer Science \& Engineering Desh Bhagat Engineering

\section{ACKNOWLEDGEMENT}

College, Moga) for critical discussion as well as constant help during the present study. The constant encouragement provided by Dr. Gurjeet Singh Associate Professor Department of Computer Science \& Engineering Desh Bhagat Enggineering College, Moga) for encouraging towards the new intrusion techniques of cross layer based.

\section{REFERENCES}

[1]. B.Mukherjee, L.T.Heberlein, And K.N.Levitt (1994) “Network Intrusion Dtetction”, leee Network,May/June pp 8-10.

[2]. Dasgupta, D., et.al. (2002). Cougaar Based Intrusion Detection System (Cids). Cs Technical Report No. Cs- 02- 001, February 4.

[3]. Debar, H., Dacier, M. And Onespi, A. (1999). "Towards A Taxonomy of Intrusion-Detection Systems". Computer Networks, 31, Pp. 805-822.

[4]. Denning D., (1987) "An Intrusion-Detection Model”, IEEE Transactions On Software Engineering, Vol. Se-13, No. 2, Pp.222-232.

[5]. Thamilarasu, A. Balasubramanian, S. Mishra, R. Sridhar( 2005), "A Cross-Layer Based Intrusion Detection Approach For Wireless Ad Hoc Networks," Proc. IEEE International Conference On Mobile Adhoc And Sensor Systems Conference. pp.861,

[6]. Jeyanthi Hall (2005) "Enhancing Intrusion Detection in Wireless Networks Using Radio Frequency Fingerprinting” IEEE Transactions on Dependable And Secure Computing 12, July. Pp 18-22.

[7]. Lim, Y, T. Schmoyer, J. Levine and H. L. Oonen. June (2003). "Wireless Intrusion Detection and Response." In Proceedings Of The 2003 IEEE Workshop On Information Assurance United States Military Academy, Ny: Onest Point. Pp 22-26.

[8]. Rakesh.S, (2010) "A Novel Cross Layer Intrusion Detection System in MANET "24th Proc. IEEE International Conference on Advanced Information Networking and Applications. Pp 38-48.

[9]. S.Madhavi,(2008)"An Intrusion Detection System In Mobile Adhoc Networks" International Journal of Security and Its Applications Vol. 2, No.3, July. Pp 11-17.

[10]. Shafiullah Khan (2010) "Framework for Intrusion Detection in IEEE 802.11 Wireless Mesh Networks", The nternational Arab Journal of Information Technology, Vol. 7, No. 4, October.pp 50-55

[11]. Zhang, Y and W. Lee, (2000)." Intrusion Detection In Wireless Ad-Hoc Networks. "In Proceedings Of The Sixth Annual International Conference On Mobile Computing And Networking, Boston: Massachussetts, August 6-11, pp 26-31. 